ÉGYPTE

monde arabe

\section{Égypte/Monde arabe}

23| 1995

Géographies de l'Égypte 2

\title{
Le Grand Caire. Étude de géographie urbaine
}

Traduction : Mona Akouri

\section{Gamâl Hamdân}

\section{(2) OpenEdition}

Journals

Édition électronique

URL : https://journals.openedition.org/ema/977

DOI : 10.4000/ema.977

ISSN : 2090-7273

Éditeur

CEDEJ - Centre d'études et de documentation économiques juridiques et sociales

Édition imprimée

Date de publication : 30 septembre 1995

Pagination : 195-236

ISSN : 1110-5097

Référence électronique

Gamâl Hamdân, «Le Grand Caire. Étude de géographie urbaine », Égypte/Monde arabe [En ligne], 23 | 1995, mis en ligne le 08 juillet 2008, consulté le 07 juillet 2022. URL : http://journals.openedition.org/ ema/977; DOI : https://doi.org/10.4000/ema.977

Ce document a été généré automatiquement le 7 juillet 2022

Tous droits réservés 


\title{
Le Grand Caire. Étude de géographie urbaine
}

\author{
Traduction : Mona Akouri
}

Gamâl Hamdân

\section{Présentation}

1 Le 17 avril 1993, le géographe et historien Gamâl Hamdân, né à Qaliûb (Delta) en 1928, disparaissait tragiquement dans l'incendie de son appartement au Caire. Personnalité singulière et contestée, il avait démissionné en 1963 de l'université du Caire, où il avait enseigné pendant une dizaine d'années après avoir obtenu en Angleterre son $\mathrm{PhD}$ de géographie. Il ne s'agissait pas seulement d'une rupture avec le milieu universitaire : le géographe se retirait également de toute vie sociale et entrait dans une sorte de réclusion volontaire qui allait être des plus fécondes.

2 L'œuvre de G. Hamdân marque un tournant méthodologique concernant le rapport entre la géographie, la sociologie, l'histoire et la stratégie. Un ouvrage surtout l'a rendu célèbre, Shakhsiyat Masr (La Personnalité de l'Égypte), ensemble de plus de 4.000 pages représentant la somme de tout ce qu'il a écrit par ailleurs et qui lui a demandé dix ans de travail. Il n'est pas impossible, aux dires de l'auteur lui-même, que cette œuvre titanesque ait nuit à ses autres ouvrages en polarisant l'attention sur elle.

3 Le sommaire de «La Personnalité de l'Égypte », paru dans la Lettre de l'OUCC n 36, peut permettre au lecteur de situer ces chapitres dans le champ général de l'oeuvre. Ce plan général, apparemment très «classique» (géographie physique, humaine, puis économique, se terminant par une synthèse régionale sur la « civilisation » de l'Égypte) masque en réalité d'incessants allers-retours entre l'Homme et le Milieu tout au long de l'œuvre: ainsi, les problématiques des sciences sociales ne sont jamais absentes, y compris dans les chapitres consacrés à la géologie. En réalité, utilisant un procédé rhétorique fondé sur la récurrence, l'auteur construit une véritable spirale à laquelle chaque partie, chaque chapitre, chaque exemple - à la limite de la redondance ajoute une pierre supplémentaire.

4 En mars 1969, les éditions Dâr al-Hilâl ont publié, à l'occasion du millénaire de la capitale égyptienne, la traduction du «Caire» de Desmond Stewart, réalisée par 
l'écrivain égyptien Yahia Haqqi et précédée d'un texte de Gamâl Hamdân. C'est ce dernier que nous présentons dans les pages qui suivent. Il s'agit de la première étude de son auteur sur Le Caire. Il nuancera par la suite sa pensée, notamment en ce qui concerne la planification de la partie la plus ancienne de la capitale; il la qualifie ici d'informelle tout en concédant qu'elle répond à une logique dont la source est aujourd'hui tarie, et qu'elle a cédé le pas aux nouveaux concepts de l'urbanisme. Il faut, bien entendu, resituer cette introduction dans le contexte de l'époque à laquelle elle a été écrite.

Elements de bibliographie

1953

The Population of the Nile Mid Delta, Past and Present: a Study in Dialectical Integration in Regional Economy, PhD, Reading University (G-B)

1958

Etudes à propos du monde arabe (Le Caire)

1959

La croissance et la distribution des habitants en Égypte

Types d'environnement

1960

"Some Aspects of the Urban Geography of the Khartoum complex », Bulletin de la Société de Géographie d'Égypte

1961

Studies in Egyptian Urbanism

« Notre planification administrative au regard de notre administration locale »,

Le miroir des sciences sociales (juin)

1962

« Pattern of Medieval Urbanism in the Arab Worid », Geography (avril)

1963

La ville arabe

La colonisation et la libération du monde arabe

1964

«Sizes of African capital », Bulletin de la Société de Géographie d'Égypte

Le pétrole arabe; une étude de géographie humaine

"Vers une école arabe de géographie », Le Miroir des sciences sociales (décembre)

"Capitals of the New Africa », Economic Geography (juillet)

1966

La nouvelle Afrique : une étude à propos de la géopolitique

1967

Les juifs, anthropologie

1968

La stratégie de la colonisation et de la libération 
y figurerait certainement. (. Et si l'on recensait les plus anciennes capitales du monde, on découvrirait dans Le Caire leur ancêtre à toutes. En tout cas, rares sont les villes qui peuvent, comme Damas, lui disputer ce rang. (...) En outre, il n'est à notre sens aucune capitale qui jouisse dans son pays d'un tel poids culturel, d'une pareille influence politique, d'un rayonnement intellectuel et national aussi fort, d'une centralité sans doute dominatrice. Les capitales sont-elles des entités qui incarnent le mieux l'âme, la substance d'un pays, creuset où fusionnent ses éléments régionaux ou bien sont-elles, par leur nature, nécessairement cosmopolites et, accueillant des communautés étrangères, forment-elles ensemble une entité particulière qui les rend plus proches l'une de l'autre qu'elles ne le sont vis-à-vis de leurs pays? Quelque réponse que l'on apporte à cette question, le cas du Caire n'est pas susceptible de controverse. Cette capitale polarise l'âme du pays, en symbolise l'essence du point de vue culturel, matériel, géographique et historique, et cela comme nulle autre.

50 Gorgé d'histoire, Le Caire en a fait des réserves. Chaque pierre en est imprégnée. Chaque fragment y porte l'empreinte de l'homme. Cette ville, immense demeure collective, bloc de constructions sans pareil dans toute l'Égypte, est un artifice, une édification considérable dont l'architecte et l'habitant est l'Égyptien et qui représente, en cela, la surface la plus «humaine » du paysage civilisé, le point du territoire où culminent les traces de l'homme et qui s'éloigne d'autant du paysage vierge et anonyme de la vallée.

51 Malgré cela, Le Caire est malheureusement la capitale qui a le moins fait l'objet de recherches urbaines récentes. Certes, les textes - académiques ou autres - consacrés à cette ville ne manquent pas; mais ils restent centrés sur l'histoire en général, sur celle du peuplement en particulier, ou relèvent de l'archéologie ; on peut y ajouter les récits « d'amateurs de villes », voyageurs, écrivains, journalistes, étrangers surtout. 

bien définis, société complexe se mouvant, s'entrechoquant dans un "contenant» géographique aux contours nets, au relief apparent, quant aux recherches en sociologie et géographie urbaines, en morphologie, sur la structure fonctionnelle du Caire, son milieu humain, la croissance de sa population, son habitat rampant et ses régulateurs, ses transports, ses problèmes de circulation, sa topographie sociale, le territoire de la ville et ses limites, les plans d'avenir et leurs indices..., quant à tout cela, donc, nous nous trouvons devant un vide inquiétant, un terrain quasi vierge, demeuré inexploré depuis la première et dernière tentative sérieuse entreprise dans ce domaine, à savoir l'étude de Marcel Clerget ${ }^{1}$ datant des années trente, et que la croissance explosive de la capitale a plus ou moins reléguée dans les coins des bibliothèques réservés à l'histoire ancienne.

Il est peut-être utile pour un Cairote, un Égyptien ou un Arabe d'avoir en tête, rassemblant les morceaux dispersés de leur ville et de ses abords, une carte simplifiée, un aperçu qui, tout en confirmant leurs notions globales, complète leur expérience quotidienne.

Le texte qui suit se veut le prélude à une recherche en géographie urbaine qui étudierait le milieu naturel où se situe Le Caire, en examinerait la croissance à travers les phénomènes apparents et sous-jacents, déterminerait les fonctions et la répartition de ses habitants selon leur appartenance sociale, etc.

Le Grand Caire : le territoire, la situation

55 Le territoire est un vaste ensemble géographique déterminé par des rapports spatiaux et des caractères régionaux dépassant de très loin les limites de la ville, et pouvant même atteindre les dimensions de tout un continent. C'est donc un concept qui varie avec les époques et il est, dans l'histoire, peu de territoires qui puissent relever d'un principe définitif. Quant à la situation, c'est tout simplement la portion locale de territoire sur laquelle se trouve directement érigé le bloc de constructions; elle ne change qu'avec la disparition du corps même de la ville et son déplacement vers une autre parcelle de territoire.

Le Caire se situe dans un territoire unique, tant par rapport à l'Égypte que par rapport à d'autres pays. C'est à la pointe où s'effectue la jonction entre le Delta et la HauteÉgypte, au nœud de la vallée, en son "nombril », que ce trouve ce territoire, foyer permanent autour duquel les capitales ont toujours décrit leur orbite, changeant peutêtre de position mais sans s'en écarter, sauf en des périodes fugaces et que l'on pourrait qualifier d'anomalies dans l'histoire nationale. Il est semblable en cela aux rives du Tigre et de l'Euphrate, en Irak, où se sont succédé les capitales, de Babylone à Bagdad, ou à la pointe du pays tunisien s'ouvrant sur les deux Méditerranées [occidentale et orientale] où se sont reproduites ou réincarnées Carthage et Tunis.

Le cadre territorial du Caire est donc cette portion de l'Égypte située au confluent des deux bras du Nil et à la jonction des deux déserts, lieu dont le pays semble avoir fait son point de ralliement. C'est pourquoi la capitale, à travers les siècles, y accomplit une trajectoire sans toutefois quitter son champ magnétique, depuis la Memphis pharaonique (actuellement dans la région d'al-Badrashayn), Onou ou Héliopolis (aujourd'hui 'Ayn Shams et Masr al-Gadîda), [la forteresse de] Babylone (Masr alQadîma), Fustat l'arabe, puis al-'Askar et al-Qatâ'iya d'Ibn Tulûn, jusqu'au Caire 
fatimide : autant de villes qui, toutes, représentent les divers maillons d'une chaîne géographique ou d'une lignée territoriale unique.

Si la capitale s'est, plus d'une fois, égarée dans un cadre territorial différent, ainsi Thèbes (Louxor) dans l'extrême sud, puis Avaris, la base des Hyksos, dans l'est du Delta, et enfin l'Alexandrie ptolémaïque et romaine, c'est que la première fut érigée à une étape donnée de la formation de l'État égyptien, tandis que la deuxième était une anomalie issue d'une invasion étrangère; quant à la troisième, ce fut le fruit de la colonisation du territoire par un empire maritime situé sur l'autre bord de la Méditerranée, et elle ressembla un moment à une île étrangère de l'archipel grec, transplantée, politiquement et humainement, sur la côte égyptienne. Le transfert de Memphis à Fustat est une transition importante dans l'orientation politique et topographique : ce déplacement s'est opéré de la rive ouest du Nil vers la rive est, et montre que Memphis, dont le contact avec le delta était presque aussi aisé qu'avec la Haute-Égypte - où les terres agricoles occupaient la rive ouest -, avait plutôt une vocation locale.

Fustat, quant à elle, présentait davantage d'affinités avec les perspectives d'une conquête arabe qui visait l'ouverture vers le monde extérieure et la continuité territoriale, le calife 'Umar ayant formellement prescrit à Amr Ibn al-'As, son chef militaire, "d'éviter toute séparation fluviale entre lui et les musulmans ». Amr choisit donc Fustat au lieu d'Alexandrie ou de Gîza, comme certains le lui avaient proposé. Ainsi, la configuration de Fustat faisait davantage penser à celle de Kûfa et Basra en Irak, s'étalant en éventail autour du cap de la péninsule arabique. Toutes trois sont situées à la limite de vallées désertiques qui, partant d'elles ou de leurs environs, finissent sur les rives d'un grand fleuve, mais sans le traverser.

C'est là également que Gîza (Jîzâ) commença à jouer son rôle de tête de pont. Son nom vient de ijtiyâz ("passage" ou "traversée ») et de majâz ("passage», " détroit", "goulet »), c'est-à-dire trait d'union entre la capitale et la Haute-Égypte. Elle hérita de l'apparence de Memphis - de l'apparence seulement - et garda ainsi, jusqu'au début de ce siècle, l'aspect d'un petit maillon rigide. Dans ce contexte, l'île de Rôda semblait un demi-pont naturel entre Gîza et Fustat, que prolongeait une autre moitié, flottante cette fois, constituée de vaisseaux amarrés.

61 Rappelons ici que Fustât, située aujourd'hui à la limite sud du complexe urbain du Caire, présentait alors un point d'accès étroit qui permettait de passer aisément entre les deux rives, à une époque où le fleuve représentait un obstacle non négligeable pour le transport. En effet, la rive est du Nil n'offrait pas le contour que nous lui connaissons aujourd'hui mais partait du voisinage de Fustât puis déviait fortement en direction du nord-est jusqu'au centre du Caire actuel, de sorte qu'une bonne partie de la ville, sans doute près du tiers, était immergée.

Ainsi, la rive est n'était pas celle que nous connaissons aujourd'hui, augmentée du territoire qui s'est formé peu à peu au fil des siècles; l'étendue en était plus restreinte, le triangle ouest s'étant formé à partir des sédiments limoneux déposés par le fleuve en constante dérive vers l'ouest, perpétuant un mouvement que l'on appelait la «migration du Nil vers l'ouest». Quant au territoire dégagé des eaux par le retrait du fleuve, il ne fut pas immédiatement utilisable, car le sol était imbibé, les lacs et marécages y abondaient et il fallut attendre des siècles de formations sédimentaires et de solidification avant qu'il fût propre au peuplement. Dans la zone d'al-Azbakiyya, par 
exemple, le terrain ne devint stable qu'à l'époque fatimide, et dans celle de Bâb al-Lûq, à l'époque ayyoubide seulement.

Nous pouvons maintenant imaginer globalement la situation du Caire. Sur la rive est s'étendent des chaînes de collines qui se rapprochent du fleuve au sud et s'en éloignent vers le nord : c'est le Gabal al-Muqattam, que prolonge al-Gabal al-Ahmar au nord, près de 'Abbasiyya. Plusieurs collines secondaires apparaissent vers l'ouest, à l'extérieur de la chaîne : ce sont, du sud au nord, les buttes de 'Ayn al-Sîra, Zaynhum et Qat' al-Mar'a.

À cet endroit, on remarque que la rive est, qui s'ouvre en éventail au nord, se rétrécit au sud; sa surface s'incline au fur et à mesure que l'on va du désert vers le fleuve. Autrement dit, le secteur est de cette rive est élevé alors que le terrain est bas dans le secteur ouest (le nom de Bulâq dérive d'ailleurs du mot balâq, qui signifie «terre basse »). De plus, le premier est de formation ancienne, alors que la formation du second est de plus en plus récente au fur et à mesure qu'on se rapproche du fleuve. Sur la rive ouest, par contre, il n'existe pas de rempart de collines; les terres agricoles s'étendent jusqu'au désert, et si le terrain là aussi s'incline, ce n'est pas en direction du fleuve mais du désert ; la pente est douce, toutefois : son angle se mesure en pouces et non en dizaines de mètres, comme c'est le cas sur la rive est. Mais elle est perceptible à l'œil nu, comme on peut le noter en regardant vers Mît Oqba depuis le pont de Zamalek.

Ainsi la rive occidentale présente-t-elle une surface plane là où seules s'étendaient, par le passé, les terres agricoles, alors que la rive orientale, en pente, est le lieu d'aboutissement de plaines désertiques el de collines que surprennent les torrents en hiver, comme peuvent en témoigner les habitants des quartiers de 'Abbasiyya ou de Gamaliyya, quartiers dont les rues inclinées se transforment parfois en canaux. Et tandis que les rues de la rive occidentale - sauf celle menant aux Pyramides - sont horizontales et d'un niveau égal, le secteur est de la rive orientale présente des rues en pente raide, presque en escalier, qui font penser aux villes de montagne du Bassin méditerranéen.

66 S'il fallait, pour conclure, commenter cet emplacement, qu'en dirions-nous? La situation du Caire présente des avantages évidents. Le fleuve et les collines entourent sur trois côtés la rive est, qui ne s'ouvre qu'au nord. La proximité des collines orientales, avec leurs carrières, permet à la ville de se fournir en matériaux de construction; quand au fleuve, l'argile de ses rives procure à la ville la matière première pour la fabrication des briques. Les zones élevées du secteur est, éloignées du fleuve, en sont dédommagées par la salubrité de l'air sec et vivifiant qui y circule, alors que la rive ouest jouit de la fraîcheur du front fluvial. Enfin, en cette région, les îles prolifèrent $\mathrm{du}$ fait du changement de niveau des alluvions lorsque s'opère la transition de la vallée étroite au vaste delta. Ces multiples îlots constituent d'importantes bases, tant pour la traversée du fleuve que pour la croissance de la ville.

La croissance du Caire : ses limites et ses axes

Dans ce cadre naturel favorable, nous pouvons suivre le mouvement de la ville à travers l'histoire, depuis la conquête arabe. À l'extrême sud, voisinant avec le fleuve et la colline, Fustât était une ville essentiellement guerrière qui voyait en cette dernière un rempart naturel. C'était donc une "acropole», c'est-à-dire une ville située sur une hauteur (Desmond Stewart va jusqu'à comparer la mosquée Ibn Tûlûn au Parthénon d'Athènes!). Lorsqu'on bâtit al-'Askar au nord-est de Fustât, puis al-Qatâ'iya, également orientée vers le Gabal Yushkur, et enfin al-Qâhira - à l'origine cité royale, interdite - cela ne modifia en rien le caractère essentiellement militaire de l'acropole. 
Ces cités se tenaient au pied de collines situées à l'est et consolidaient ces remparts naturels par une deuxième ligne de défense, les enceintes successives de la ville. Le seul fait notable est qu'à partir d'un emplacement initial au sud, elles se déplaçaient vers un autre, plus au nord.

Il est intéressant de constater, puisqu'il est question des villes fortifiées et de leurs enceintes, que l'Égypte représente dans ce contexte une exception remarquable, tout comme Le Caire en représente une autre par rapport à l'Égypte... Au Moyen-Âge, au temps des régimes féodaux, la ville fortifiée était de règle : il s'agissait de la protéger tant des dangers externes que des luttes féodales internes. Trois pays dans le monde ne connurent pratiquement pas ce modèle en raison de la protection naturelle dont ils

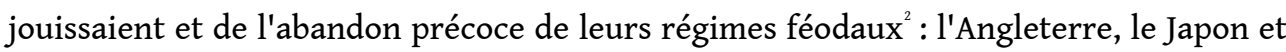
l'Égypte. Ces trois pays sont des îles, au sens propre ou au sens figuré : ils sont séparés du continent par une mer d'eau ou de sable. Le désert est le rempart naturel de l'Égypte, indiquait Louis Memford; ce qui n'est pas tout à fait le cas pour Le Caire. De par sa position et son importance, la capitale a toujours été exposée aux dangers venant de l'extérieur, ou menacée par les dissensions internes. Les remparts constituaient donc pour elle une nécessité stratégique; les murs d'enceinte se multiplièrent et s'élargirent avec le développement de la cité, alors que les villes de province, à l'exception de certains ports situés aux confins du pays, ignoraient murs et remparts.

69 À des étapes ultérieures, la ville, après s'être développée vers le nord, commença à s'étendre vers l'ouest. Avec l'extension des terrains limoneux aux dépens du fleuve, l'exploitation agricole, et plus tard le développement du bâti, allaient également progresser dans cette même direction. La ville amorça peu à peu un glissement vers les paliers inférieurs: alors que, craignant la proximité du fleuve, elle s'agrippait aux flancs et sommets des collines, elle se mit à changer d'aspect quand fut écartée la menace d'une crue ou d'une extension du lit du fleuve: de ville haute, d'acropole suspendue, elle se transforma en ville riveraine et plane. Se libérant de l'étau montagneux, la cité échappait à la captivité des murailles.

70 La zone de peuplement se développa donc en suivant, globalement, un axe nord-ouest. Tel est le mode d'extension du Caire à travers l'histoire, selon un mouvement continu et un rythme constant malgré les périodes d'immobilisme et de repli.

71 Jusqu'à l'expédition française, l'axe formé par al-Husayniyya, Bâb al-Sha'riyya et Bûlâq représentait la limite septentrionale de la ville sans pour autant que la zone sud fût entièrement peuplée et bâtie : elle présentait de vastes zones vides. Vers le nord, le peuplement était diffus. C'est Muhammad 'Ali qui, dépassant cette limite, opéra une percée vers Shubra, de même que le khédive 'Abbas fonda 'Abbasiyya dans le prolongement d'al-Husayniyya. C'est encore Muhammad 'Ali qui ouvrit la voie vers Garden City pour y bâtir les luxueuses résidences de sa famille ; le quartier d'Ismalliyya ne fut entamé qu'au temps d'Isma'îl, et celui d'al-Tawfiqiyya au temps de Tawfiq.

Le développement décisif du secteur englobant al-Faggâla, al-Dahir, Ghamra et alSakakîni, au sud de la ligne de métro et de la gare du Caire, n'a eu lieu qu'après 1900. Plus récente encore, bien sûr, est l'extension nord-est à partir d'al-Damardâsh et de Manshiyyat al-Sadr en passant par al-Qubba, tous secteurs compris, et Manshiyyat alBakri où l'on trouve deux ramifications : l'une en direction du nord vers al-Zaytûn, alHilmiyya, al-Matariyya puis 'Ayn Shams, l'autre en direction du sud vers Masr alGadîda. Ceci est également vrai pour le développement de la zone nord à partir de Rôd 
al-Farag jusqu'à al-Sâhif et Shubra (tous secteurs compris : af-Hadâ'iq, al-Khayma, alMizallât et al-Balad\}.

Sur la rive ouest, Gîza garda des proportions modestes jusqu'au début du siècle et continua à progresser lentement vers le nord, formant un ruban qui allait en s'élargissant. Durant la deuxième guerre mondiale et par la suite, elle allait atteindre Imbâba à travers Doqqi et 'Agûza; sa largeur devint alors comparable à celle du quartier d'al-Sâhil sur la rive est. Le développement de la rive ouest, exception faite du centre commercial (al-Bandar) de Gîza, est récent par rapport à celui de la rive opposée.

Par conséquent, non seulement la croissance de la ville, sur les deux rives, s'effectue vers le nord, mais encore - ce qui n'est pas moins significatif - cette croissance s'interrompt brutalement au sud, également sur les deux rives. Masr al-Qadîma n'a pas franchi ses limites stables aux environs de Athar al-Nabî; la vieille ville de Gîza (le centre commercial) non plus. Et si Ma'âdi et Hilwân, sur la rive orientale, se sont développées récemment - Hilwân depuis Isma'îl, comme station thermale, et Ma'âdi avec l'expansion et la consolidation de la colonie britannique -, ce ne sont que des banlieues séparées de la ville, ce qui n'invalide pas notre constat mais le confirme. On peut en dire autant pour la région des Pyramides, qui se développe de façon linéaire à la lisière de la ville.

75 En conclusion, il apparait que les limites sud du Caire sont stables alors que celles du nord sont variables, dynamiques. La différence entre les deux noms, Masr al-Qadîma (l'ancienne), à l'extrême sud, et Masr al-Gadîda (la nouvelle), à l'extrême nord, résume éloquemment l'histoire et le mouvement qui s'est dessiné à l'intérieur de cet énorme complexe urbain.

Toutefois, l'emplacement du Caire n'explique pas à lui seul l'opposition entre le nord et le sud. Car il ne fait pas de doute que le Delta, avec sa richesse agricole, sa fertilité, son ouverture par les ports et les échanges commerciaux avec l'extérieur, constitue un pôle d'attraction pour la capitale, dans la mesure où, plus facile d'accès et mieux organisé pour l'écoulement des marchandises à l'extérieur, il est mieux à même de fournir à son industrie des matières premières et à ses habitants de la nourriture. On peut même dire que la progression du Caire vers le nord est en définitive un lointain reflet de l'attirance exercée sur la capitale par Alexandrie et par Suez.

Si cette opposition entre le nord et le sud est criante, elle existe tout autant entre l'est et l'ouest. À l'est s'élève la muraille du Muqattam, étouffant toute possibilité de croissance ; même aujourd'hui, l'urbanisation du plateau du Muqattam ne représente qu'une tentative symbolique. Côté ouest, la ville a colonisé le fleuve même - c'est-àdire les deux îles de Gazîra et de Rôda - puis elle l'a traversé (...), de sorte que le complexe urbain dans son ensemble figure une cité déployée sur deux rives, à cheval sur le fleuve.

Il est probable qu'à l'avenir, la croissance de la rive ouest dépasse celle de la rive opposée dans la mesure où la première joue actuellement un rôle pionnier et constitue le ressort principal de l'extension de la capitale. En d'autres termes, si la croissance est aujourd'hui plus forte vers l'axe nord, elle pourrait, en quelques décennies, se déplacer vers l'ouest. De nos jours, la rive ouest a gagné en profondeur, atteignant Bulâq alDakrûr au sud et Mît 'Oqba au nord, et continuera probablement de progresser en direction de l'artère constituée par la ligne de chemin de fer liant la Haute et la BasseÉgypte. 
(laquelle se trouvait jadis en face de Memphis), pointe qui elle-même remonte sans arrêt vers le nord. Ou encore, comme si elle entraînait à sa suite toute l'Égypte moderne : le développement, en effet, s'est ralenti à l'extrême sud de la Haute-Égypte, surtout depuis la construction du haut barrage, alors qu'il croît dans le nord du Delta, notamment avec la bonification des terres désertiques, qui bientôt étendront leurs cultures jusqu'à la mer. C'est enfin comme si elle symbolisait le déclin relatif de la Haute-Égypte qui, en comparaison avec le Delta, ne fournit plus aujourd'hui que $38 \%$ $\mathrm{du}$ revenu de l'agriculture égyptienne. Cela nous fait entrevoir une autre similitude entre la croissance du Caire et l'étendue des terres cultivées en Égypte. Si l'on regarde une carte du Caire, on remarque tout de suite sa forme évasée. C'est une ville plus longue que large : $13 \mathrm{~km}$ de longueur sur $7 \mathrm{~km}$ de large tout au plus, avec une largeur moyenne bien inférieure et quasi étranglée à l'extrême sud. Et alors que le Nil constitue l'axe nord-sud, le tracé reliant Masr al-Qadîma à Masr al-Gadida s'en écarte nettement. Le front qui avance vers le nord ne suit pas une ligne régulière mais se creuse en son milieu, formant une fourche : le tronçon de Masr al-Gadîda-'Ayn Shams au nord-est, en bordure du désert, et celui de Shubra-Rôd al-Farag au nord, longeant le Nil ; les deux parties sont séparées par une vaste étendue de terres agricoles.

81 Le Caire a donc la forme d'un éventail sur lequel apparaissent les caractéristiques du lieu et son relief original, un éventail ouvert, étalé, avec sa tige au sud, qui évoque immédiatement, bien qu'en tout petit, la forme du Delta lui-même. Les deux coulées précédemment évoquées, qui progressent vers le nord, complètent la comparaison: elles font penser aux deux bras du Nil, celui de Damiette et celui de Rosette. Si nous ajoutons la « tige » tronquée du sud, à la croissance intermittente, qui passe par Ma'âdi et Hilwân, l'aspect du Caire se rapproche de celui de l'Égypte, où le sud dessine la tige, quelque peu fragile, de l'éventail du Delta. Non seulement notre capitale englobe l'entité humaine de l'Égypte, mais elle récapitule aussi, comme le cartouche d'un blason, sa forme géographique.

Qu'en est-il de la croissance verticale du Caire, qui a évolué en synchronie avec sa croissance horizontale? L'histoire de la ville ne se limite pas à l'extension des pourtours, elle passe également par une densification interne. Il fut un temps où les espaces construits du Caire étaient traversés de grands vides désolés, d'espaces à l'abandon. Jusqu'au début du XIXe siècle, le corps de la ville était diffus, disjoint, entaillé, puis il se resserra peu à peu, cicatrisa progressivement. Alors qu'à la périphérie, les villas poussaient au milieu des champs, celles du centre se métamorphosaient en immeubles qui jouaient du coude, s'empoignaient, luttaient de vitesse pour gagner en hauteur tels les arbres d'une forêt se débattant pour s'ouvrir un accès au soleil. La ville était tout près d'étouffer, cherchait en vain quelque poumon vert ou espace découvert. Qui observe aujourd'hui, sur une carte du Caire, l'espace bâti, croira à tort y repérer des vides non exploités, telles ces saillies formées par les buttes

Égypte/Monde arabe, 23 | 1995 
de 'Ayn al-Sîra, Zaynhum et Qat' al-Mar'a, à l'est de la ville. Ce sont en réalité les limites des régions évoquées, et elles ne font que séparer la cité des vivants de celle des morts. Quant à l'espace bâti proprement dit, il forme un bloc compact et continu.

Pour conclure notre propos sur le développement de la capitale, il nous faut marquer un arrêt et tenter de répondre à cette question; qui a lâché les rênes d'une ville qui est devenue, depuis le siècle dernier notamment, comme le génie échappé du flacon? La ville médiévale n'a pas débordé l'espace modeste, limité, qu'elle occupait à l'est; elle n'est sortie de sa coquille, historiquement et géographiquement, que vers la fin du Moyen-Âge, encore qu'avec timidité. Ce n'est qu'au siècle dernier qu'elle a amorcé un mouvement d'extension nouveau, en direction du fleuve, à un rythme soutenu au cours des premières décennies de ce siècle. Et c'est seulement après la deuxième guerre mondiale qu'elle explosa, véritable raz-de-marée qui, depuis la révolution, a atteint une vitesse et une impétuosité jamais connues auparavant. Nous pouvons distinguer, dans l'histoire de la cité, trois étapes ; l'étape embryonnaire, l'étape de la formation et celle de l'explosion.

La surface occupée par Le Caire s'est peut-être accrue, au cours du siècle précédant la deuxième guerre mondiale, plus qu'elle ne l'a fait tout au long du millénaire qui a vu naître la ville arabe, c'est-à-dire pendant l'étape que nous appelons embryonnaire, alors que sa croissance, au cours du dernier quart de siècle, dépasse celle qui fut la sienne tout au long du siècle antérieur. La capitale a échappé à la tutelle patriarcale de la montagne, s'est étendue du Muqattam aux Pyramides, du désert au désert, de Hilwân à Shubra al-Khayma, et après s'être cantonnée aux limites rigoureusement tracées, telles une figure géométrique, par le mur de la ville, elle a débordé sur les terres cultivées puis les a englouties. On en constate aisément les conséquences en examinant les recensements : la ville qui, au début du règne de Muhammad 'Ali, comptait un quart de million d'habitants, et à la fin de son règne, un tiers de million, a dépassé aujourd'hui cinq millions d'âmes.

Demandons-nous à nouveau qui a lâché la bride à ce terrifiant développement. Il existe deux facteurs, régulateurs ou moteurs, dont aucun ne constituerait à lui seul une explication, sauf pour une période limitée. Ce sont la situation de la capitale et les communications. Sa croissance, à l'étape embryonnaire, correspond à l'élargissement, du côté du fleuve, du territoire environnant. Mais rien ne peut expliquer les deux étapes suivantes, si ce n'est la révolution des communications. Jusqu'à Muhammad 'Ali, ce sont les bêtes de somme qui servaient de moyen de transport à l'intérieur de la ville, et les embarcations à voile qui la reliaient à l'extérieur. Le mouvement humain avait le souffle court, d'où l'extension nécessairement réduite de la ville. Puis commença à se dérouler la chaîne historique ; des bêtes de somme, on passa aux fiacres, puis aux lignes régulières "Suarès", au tramway et enfin aux voitures, privées ou publiques. Les limites de peuplement du Caire étaient, à chaque étape, fonction du moyen de transport en usage.

Une deuxième question, découlant de la première, s'impose : peut-on considérer celte croissance comme un phénomène tout à fait sain ? Evolue-t-elle selon les tracés les plus adéquats, les orientations les plus rationnelles? Ne nous arrêtons pas ici à l'inflation démographique d'une capitale dotée de 5 millions d'habitants dans un pays qui en compte 30 millions ou davantage. Ne parions pas non plus de "tumeur", terme par lequel Cobbet désignait Londres à l'époque industrielle. Le Caire souffre probablement de «mégapolisme» tout comme l'Égypte elle-même souffre d'une démographie 
excessive. Mais ce qui est plus grave que ce développement sauvage, c'est ce trait persistant, chronique, qui fait soupçonner une prolifération cancéreuse par essence.

L'allusion vise certainement ici l'extension de l'espace bâti sur les terres agricoles, ceci dans un espace géographique captif et affamé de terre arable. Nombreux sont les habitants du Caire qui se souviennent avoir vu,au cours de leur vie, les milliers de feddan cultivés de Shubra et Gîza, ou les routes et les lignes du tramway courant à travers les plantations denses, les vergers, les potagers et les jardins qui, par la suite, allaient disparaître progressivement, certains continuant de résister comme de vaillants îlots au milieu de la marée montante. Tout cela s'est aujourd'hui transformé en une étendue compacte de constructions mettant en déroute les plantations, les exilant vers de lointains horizons. Si ce n'est pas le cas pour le tronçon allant vers Masr al Gadîda, cela l'est malheureusement pour celui qui se dirige vers 'Ayn Shams, où l'espace bâti gagne sur les terrains agricoles au lieu de les longer, recourt à l'agression plutôt que d'entretenir des rapports de bon voisinage.

La ville dévore ses habitants, dit-on, mais ici, elle dévore aussi le territoire; de la famille des rongeurs de terres agricoles, elle en a la voracité. Il est temps que le sable devienne le lot des bâtiments, et la terre grasse celui de l'agriculture ! L'axe sablonneux le plus propice à la construction se trouve au nord-est du Caire, dans le prolongement d'al-'Abbasiyya, Madinat Nasr et Masr al-Gadîda. Il faudrait ensuite séparer les banlieues du corps de la capitale pour qu'elles soient bâties non pas au cœur de la vallée mais en lisière des deux déserts, notamment le long des sorties principales de la ville, sur les routes sahariennes d'Alexandrie et de Suez.

Plans et réseaux de communications

Un regard rapide sur une carte du Caire permet d'y relever trois traits caractéristiques.

Tout d'abord, deux éléments principaux se partagent la ville: d'une part, une texture informelle, spontanée, reproduisant celle des villes antiques ou même des villages égyptiens et qui, dans la capitale, représente les anciens noyaux; d'autre part, un plan géométrique qui représente l'élément contemporain, « européen », de la structure des villes égyptiennes, introduit seulement au siècle dernier. Cette dualité essentielle symbolise éloquemment la dualité culturelle de l'Égypte contemporaine où coexistent l'ancien et le nouveau, l'élément indigène et l'élément exogène.

91 Second trait: la nette prédominance de l'étendue organisée suivant une planification géométrique moderne, par rapport à la texture informelle ancienne. Cela peut paraître étrange étant donné le caractère récent de la planification géométrique régulière. En réalité, ce trait résume d'un seul coup d'œil l'histoire de la croissance de la ville moderne: on constate en effet que la plus grande partie du bloc urbain s'est essentiellement formée au cours du siècle dernier. En outre, de nombreuses transformations ont été effectuées dans les quartiers relevant de la planification ancienne, ce qui a réduit leur surface sans pour autant en effacer les vestiges.

Troisième et dernier trait : les zones à texture informelle se confinent principalement dans les limites de la ville ancienne, en particulier à l'est et au sud, bien que l'on en trouve encore, exceptionnellement, au nord et dans la zone médiane. Cela est encore plus évident sur la rive ouest : là, l'ancienne texture ne se rencontre qu'à l'extrême sud et la planification géométrique domine tout le nord. Autrement dit, l'ancienne texture correspond aux contours les plus élevés de la ville, contrairement aux zones à planification géométrique moderne. 
Ce dernier élément correspond à la règle déterminant en général le tracé de la ville égyptienne, où nous trouvons toujours un bloc ancien, au plan informel, dans le secteur sud, Juché sur une butte bombée semblable à un plat renversé, tandis que s'étend dans le secteur nord, en terrain plat, une zone organisée selon la planification moderne. Le secteur sud abrite le noyau de l'époque pré-moderne; le secteur nord représente la croissance du siècle dernier. La superficie de chacun des deux secteurs est proportionnelle à la courbe de croissance et d'extension de la ville à l'époque moderne : plus importante est la croissance, plus forte l'onde explosive, et plus le noyau ancien se rétracte par rapport à la surface du tracé géométrique moderne, et réciproquement.

Nous pouvons maintenant, à la lumière de ces indices, observer la planification du Caire avec précision. Commençons par la zone ancienne. C'est une sorte de tracé instinctif, non prémédité, s'agençant par le seul rassemblement des bâtiments. C'est, dans son essence, le tracé du village égyptien qui, s'il n'est pas dépourvu de logique - et possède même une logique géométrique - est conçu de manière approximative. L'agglomération est entourée d'un chemin circulaire mais irrégulier, suivant le périmètre, d'où partent nombre de ruelles étroites qui traversent les zones bâties pour finir au cœur du village. Là, les impasses sinueuses se ramifient, s'infiltrent plus ou moins dans un bloc de constructions apparemment informel mais, en réalité, organisé selon un embryon de plan concentrique.

On retrouve le plus souvent ce type de tracé dans les quartiers est et sud du Caire, sur la rive orientale du Nil. C'est le vieux Caire avec ses quartiers historiques traditionnels : Bab al-Sha'riyya, al-Azbakiyya, al-Dâhir et al-Husayniyya au nord, et Sayyida Zaynab, Tulûn et Sayyida Nafisa au sud, avec une résurgence à Masr al-Qadîma à l'extrême sud. Cette zone se caractérise par l'étroitesse des ruelles et des impasses dont l'extrême sinuosité est soulignée par le relief de la butte où loge la ville ancienne, mais qui courent parfois aussi en terrain plat; la densité du peuplement et de l'encombrement s'en trouve intensifiée, le tout se perd dans un lacis labyrinthique impraticable pour les moyens de transport modernes. Il a donc fallu retoucher, corriger en élargissant et même en perçant beaucoup de rues et de ruelles, c'est-à-dire en procédant à l'accouplement forcé de l'informel et du planifié, opération qui prend de plus en plus d'ampleur aujourd'hui.

Et tandis que ces quartiers semblent se perdre dans un écheveau confus, leur sont juxtaposées quasi immédiatement, à l'est comme au sud, des surfaces à la disposition géométrique régulière, précise, occupant une grande place sur la carte de la ville. Il ne faut cependant pas s'y tromper, car il s'agit de la cité des morts - tombes et cimetières diffus dans les quartiers d'al-Khalîfa, de Qayt Bey ou d'al-Ghafîr - que parcourent des rues au tracé régulier portant, comme le remarque avec étonnement Desmond Stewart, des noms et des numéros!

7 Puis nous faisons à nouveau irruption dans l'informel - avec les mêmes tentatives d'ajustement, de "chirurgie esthétique », imposées par l'administration : nous voici dans le quartier de Bûlaq, île insolite au milieu d'un ensemble planifié. Nous ne retrouvons ensuite l'informel qu'en traversant le fleuve, à l'extrême sud de la rive ouest, c'est-à-dire dans le noyau ancien de Gîza (le centre commercial) qui présente un violent contraste avec les quartiers au tracé géométrique et régulier situés plus au nord.

Pénétrant dans la zone récemment planifiée - qui occupe le reste de la capitale à l'exception de quelques îlots à la planification informelle dispersés en bordure de la 
ville, anciens villages engloutis par de récentes constructions comme Maniyyat alSarîg, quelques hameaux éparpillés au nord de Shubra et les villages d'Imbâba, Mit 'Oqba, Bulâq al-Dakrûr sur la rive ouest -, nous trouvons une image tout à fait différente, à la fois simple et complexe. La ville s'organise ici en une sorte de mosaïque illimitée constituée d'unités géométriques régulières, carrées ou rectangulaires, dont certaines vont jusqu'à former un cercle ou un polygone. Lignes bien tracées, angles droits, habitations à l'architecture similaire. La complexité de cette zone vient de ce que ces formes homogènes et rectilignes ne sont pas orientées selon un axe unique comme c'est le cas, par exemple, dans la ville américaine. Elles suivent - littéralement - des dizaines d'axes dont l'orientation diffère d'une unité à l'autre et qui font de chacune une pièce autonome comme le sont celles d'un puzzle. Voilà pourquoi nous parlons à la fois de simplicité et de complexité. Seules font exception Ma'âdi et Hilwân, dont l'orientation présente une forte unité dans toute l'aire construite, rigoureusement quadrillée et planifiée.

$\mathrm{Si}$, au Caire, les axes qui gouvernent les pièces de cet échiquier divergent totalement, il importe de savoir qu'ils n'ont pas été déterminés arbitrairement, leur orientation ayant obéi à deux facteurs fondamentaux ; le fleuve, sur lequel donne une grande partie de la façade urbaine, et les avenues principales, c'est-à-dire les artères qui traversent les quartiers et conduisent le mouvement à l'intérieur de ces derniers comme entre chacun d'eux.

Le fleuve est un vecteur d'orientation décisif, incontournable. Sur les deux rives, mais plus particulièrement sur la rive ouest, se déroulent la corniche et, au-delà, une rue principale ; elles longent ou côtoient le fleuve comme, respectivement, la rue de Gîza et la rue Qasr at-'Ayni, en en épousant fidèlement les sinuosités et les détours. Il en va de même pour les rues secondaires parallèles. Les voies transversales étant perpendiculaires aux voies longitudinales, l'ensemble du réseau connaît, d'un secteur à l'autre, une modification des orientations initiales en fonction des détours du fleuve.

Sur la rive ouest, de Doqqi à Imbâba, rien ne déroge à cette règle. Il en est de même pour la rive est, au sud de Midân al-Tahrir et le long de la voie ferrée de Hilwân; les rues longitudinales sont parallèles au fleuve, les transversales lui sont perpendiculaires. Il en est de même dans l'île de Rôda : les rues qui la traversent en longueur sont parallèles aux deux rives, et là où l'île se rétrécit, le tracé suit l'axe d'une seule rive, d'où l'apparition de portions triangulaires hétéroclites. Cette disposition est aussi évidente à Fomm al-Khalîg et à Abû-l-Su'ûd, au nord de Masr al-Qadîma, ainsi qu'au nord, à Rôd al-Farag, et sur le Sâhil en général.

102 L'incidence des rues principales sur le plan apparaît plus clairement à l'intérieur de la ville, loin de l'influence du fleuve. Là, elles deviennent comme l'épine dorsale sur laquelle s'articulent - à angle droit - les détails du plan géométrique; si elle accuse une déviation, ceux-ci la suivent et s'orientent selon elle. Pour ce qui est de la trajectoire de ces artères, elle est déterminée par leurs positions relatives entre les points stratégiques ou par les anciennes bornes du lieu, tels les canaux qui ont été comblés et transformés en boulevards et avenues principales ; c'est le cas d'al-Khalîg alMasrî (actuellement, rue Port-Saïd) et d'al-Tur'a al-Bulâqiyya.

Il existe de nombreux exemples d'une telle disposition. À Shubra, nous trouvons un axe rectiligne, la rue Shubra, et un autre, dévié, la rue al-Tur'a al-Bulâqiyya, et tous les détails du plan quadrangulaire de l'ensemble du quartier épousent l'orientation de chacun des deux axes. Mais l'exemple classique est celui du nord-est du Caire, à partir 
de Ghamra et d'al-Dhâhir jusqu'à Masr al-Gadîda et 'Ayn Shams, où l'axe directeur est représenté par la ligne de métro de banlieue. Sur toute l'étendue de cette zone, on remarquera le quadrillage régulier des rues; toutefois, les axes sont nombreux et très divergents. Mais ces unités sont à leur tour déterminées par leur point d'appui, ou plutôt leur articulation par rapport à la ligne de métro, dont la trajectoire s'infléchit selon sa destination. Il en résulte que les blocs d'habitation de la zone de Ghamra, par exemple, s'articulent sur un axe approximatif est-ouest, alors que dans la zone de Matariyya et de 'Ayn Shams, l'axe prend la direction nord-sud puis se redresse progressivement entre les deux zones, en suivant un mouvement pendulaire.

Le quartier de Zamâlek - la moitié nord de l'île - offre un cas assez pittoresque dans la mesure où s'y combinent l'ascendant du fleuve et celui de la rue principale, donnant au tracé un cachet exceptionnel. Les rues longitudinales suivent l'axe de la rue principale qui coupe l'île entre le pont du 26-Juillet et celui de Zamâlek; ainsi, les rues longitudinales et transversales se croisent à angles aigus, laissant l'espace intermédiaire se découper en formes géométriques rares telles que losanges, trapèzes, etc. En revanche, la zone située au sud de la rue qui relie les deux ponts est régie par un réseau de quadrilatères réguliers, dont les ruelles sont parallèles ou perpendiculaires à cet axe.

Il nous faut enfin évoquer un type particulier, local, de planification où prédominent non pas les tracés à angles droits mais l'enchevêtrement de courbes. Ce type d'organisation s'inspire de celui des jardins anglais; il s'agit de Garden City et de Hadâ'iq al-Qobba. Dans ces quartiers, le tracé des rues présente des arcs ou des cercles aux lignes entrecroisées, dont le centre se déplace. Autant cette planification offre une perspective architecturale grandiose dans un paysage vivifiant, autant elle provoque de problèmes de circulation. Ces deux quartiers aux dédales inextricables sont, de ce point de vue, difficiles à vivre, aussi bien pour leurs habitants que pour les autres citadins.

Si nous considérons l'ensemble des zones planifiées de la capitale, nous pouvons déduire, de la multiplicité d'orientation des axes locaux, qu'elles n'ont pas été bâties selon un plan global mais par fragments. C'est pourquoi l'articulation des quartiers les uns aux autres est souvent défectueuse ou incohérente et laisse apparaître des parcelles intermédiaires aux formes aberrantes, ou qui présentent des arêtes vives.

Certes, cette pluralité d'orientations divergentes rompt la standardisation du plan, la monotonie des quartiers et des rues, offre de nombreuses possibilités d'orientation par rapport au soleil et aux vents, ce qui permet une meilleure aération et une meilleure exposition, une alternance de la lumière et de l'ombre et, entre autres, empêche la ville de se transformer en couloirs où s'engouffreraient les vents du nord, prédominants dans la région.

Toutefois, cela ne laisse pas d'avoir une incidence négative sur le réseau de communications censé garantir l'articulation organique de la ville, et qui apparaît comme étant lui-même incohérent et disloqué ; c'est là son point faible, comme en témoignent les tentatives locales qui ont été faites de plaquer un ensemble de rues rayonnant sur des tracés géométriques quadrangulaires, aménagement qui se rapproche de ces plans concentriques aux rayons plutôt sinueux que l'on peut voir dans le centre-ville d'Ismâ'iliyya, dans !e centre de Rôda ou dans les quartiers d'al-'Agûza, alDâhir et surtout, à Masr al-Gadîda.

Tout cela n'est le plus souvent que ravaudage et subterfuge. À la vérité, Le Caire s'est développé pièces par pièces et elles ont été juxtaposées les unes aux autres à travers le 
temps, sans cadre général. Si nous ajoutons à cela le problème des zones informelles étranglées et l'énorme étendue de la capitale, nous en déduirons que Le Caire est une ville dont il est malaisé de connaître les diverses parties, où il est ardu de se mouvoir: Mais nous abordons là la question des communications et des moyens de transport de la capitale.

Malgré la présence de rues principales qui tentent de rectifier les erreurs du plan quadrangulaire pluri-axial et celles des tracés non planifiés, on ne peut parler d'une superstructure qui engloberait la totalité de la capitale : les rues et lignes de transport principales partent en effet de plusieurs noyaux. Le plus important est sans doute celui de la gare du Caire (Mahattat Masr) d'où partent les rues de Shubra au nord, Bulâq à l'ouest, al-Gala' au sud-ouest, al-Gumhuriyya au sud et enfin la rue Ramsès, la porte qui mène aux banlieues du nord-est. La place 'Ataba constitue un autre noyau : c'est là que se déverse tout le trafic de l'est de la ville, de la rue al-Gaysh à 'Abbasiyya, de la rue alMoskî-Gôhar à Gamaliyya, de la rue al-Azhar à al-Ghuriyyah et al-Darrâsa et enfin, de la rue al-Qala'a à la Citadelle et al-Khalîfa, les places Bâb al-Lûq et Sayyida Zaynab sont également le centre d'un réseau.

Cette disposition ne constitue pas pour autant un plan concentrique, même si les lignes de tramway, jusqu'à une époque récente, formaient clairement un réseau rayonnant à partir, notamment, de deux noyaux, la gare du Caire et la place Sayyida Zaynab.

Il nous faut en outre remarquer l'influence de l'emplacement des ponts sur le réseau de communication. Des deux côtés du fleuve, les deux ponts de Tahrîr et de Gala' sont le point de départ d'une gerbe d'axes; bien plus, chacun de ces deux noyaux est la véritable porte d'accès aux rives du fleuve. Il en est plus ou moins de même pour les ponts du 26-Juillet et de Zamâlek au nord, de Gîza et d'al-Malik al-Sâlih au sud. La position de ces ponts - homologues et interdépendants, passages obligés mais aussi goulots d'étranglement entre les deux rives - conditionne la trajectoire des grandes artères qui traversent la ville de part en part. Or, au Caire, l'insuffisance de grandes artères est manifeste. Et parce que Le Caire est une ville plus longue que large, les axes principaux sont des axes nord-sud, passant nécessairement par le centre-ville qui s'en trouve asphyxié. D'où l'idée de construire un boulevard périphérique reliant les quartiers limitrophes sans traverser le centre : c'est le cas de la rue Port-Saïd, la plus longue du Caire, principalement liée à la partie ancienne de la ville, et de la rue Salâh Sâlim.

113 Il apparait ici clairement que le problème des communications dans la capitale est indissociable de sa morphologie et de ses caractéristiques purement topographiques. Les deux principaux aspects morphologiques du Caire sont les suivants : d'une part, la présence d'un fleuve qui scinde la ville en deux, ce qui rend problématiques les trajets quotidiens et fait des ponts fluviaux des points stratégiques essentiels; d'autre part, la forme des quartiers frontaliers au nord de la ville, qui s'avancent en deux énormes triangles à Shubra-Rôd al-Farag et à Masr al-Gadîda-'Ayn Shams, et qui s'articulent au corps de la ville par leur sommet le plus étroit, soit, encore une fois, par des goulets d'étranglement. Cette configuration apparaît clairement dans le dernier cas : le triangle s'étire, effilé, en fer de lance, presque déconnecté sauf en un point, celui de Kubri alQubba. En chacun de ces points, le problème des communications s'intensifie jusqu'à l'asphyxie, d'autant que les quartiers limitrophes de la rive ouest et du nord de la rive est sont, à quelques exceptions près, des quartiers d'habitation plus que des quartiers de bureaux. Le problème s'aggrave de diverses façons selon la nature des quartiers: 
dans les quartiers populaires où les véhicules privés sont peu nombreux, la forte densité démographique entraîne une densité proportionnelle des transports publics; dans les quartiers à moindre densité de population, c'est la concentration des voitures privées qui s'intensifie.

Le réseau ferroviaire, à l'intérieur de la capitale, n'est pas moins problématique. Les stations de chemin de fer sont en quelque sorte à la ville contemporaine ce qu'étaient les portes de la Cité aux villes médiévales, si ce n'est qu'elles ont quitté l'enceinte pour gagner le centre. Ce sont les "entrées" de la ville, mais en son sein, comme en témoigne d'ailleurs les toponymes, "Bâb al-Hadîd " et "Bâb al-Lûq ", qui semblent vouloir nous rappeler avec insistance qu'ils se réfèrent à la fonction - et non à la situation - héritée de « Bâb Zuwayla » ou de "Bâb al-Nasr ", par exemple...

115 Les gares ferroviaires occupent des points tout à fait stratégiques dans la capitale. La gare du Caire et celle de Bâb al-Lûq sont comme les clés topographiques de la ville. C'est d'elles que partent dans trois directions, nord, nord-est et sud, les lignes nationales et celles de la banlieue. Chacune est doublée d'une station centrale d'autobus qui grouille en permanence comme une ruche et qui constitue un point de rupture, un lieu où l'on abandonne un moyen de transport pour un autre (voiture/train ou l'inverse). Toutefois, elles aggravent le problème de l'encombrement, d'autant que leurs lignes s'enchevêtrent dans le tissu urbain et étouffent le trafic, comme on peut notamment le constater le long de la ligne de métro nord-est.

116 Les gares ferroviaires, en aggravant les problèmes de circulation, ont fini par transformer la coexistence train/voiture en conflit déclaré. Les chemins de fer en sont sortis victorieux, puisque la gare routière des autobus de province a été déplacée vers le quartier limitrophe de Shubra al-Mazallât. À l'inverse, à Bâb al-Lûq, c'est le train qui semble devoir "perdre la guerre »: le projet de métro souterrain prévoit en effet de déplacer le terminus de la ligne de banlieue sud vers Kubri al-Malik al-Sâlih.

117 L'enchevêtrement de tous ces problèmes a fini par engendrer une crise chronique. La capitale, désespérant des "solutions superficielles", a recours aux «solutions souterraines ", à savoir un métro souterrain dont le plan prévoit une ligne couvrant la longueur du Caire. Les racines du problème sont néanmoins multiples. L'une d'elles, par exemple, relève d'un problème de civilisation: le tracé des rues, conçu à une époque antérieure à l'automobile et à l'industrie, souffre forcément aujourd'hui de congestion.

L'expérience des grandes capitales a prouvé que le métro ne détenait pas nécessairement le mot de la fin dans cette affaire et que les problèmes de surface ne tardaient pas à réapparaître. Londres et Paris ont leur métro depuis des décennies, New York de même, et les problèmes de circulation y demeurent chroniques. La leçon que l'on pourrait en tirer est que Le Caire a réellement besoin - avec ou sans métro d'une opération "haussmannienne » à l'instar de celle qu'a connue Paris vers 1870 ; une opération audacieuse, conçue avec imagination, sans être forcément radicale ou mutilante. Elle imposerait, sur un terrain planifié en mosaïque, une distribution concentrique rayonnant à partir de noyaux multiples d'où partiraient boulevards et artères axiales, de sorte que la canalisation des transports urbains au cœur de la ville deviendrait comparable à un fleuve ayant peu d'affluents et beaucoup de débouchés.

Il est par ailleurs nécessaire d'opérer une nouvelle répartition du travail et de l'habitat à la périphérie du Caire. La concentration du travail dans le noyau commercial central (CBD, comme l'appellent les Américains), alors qu'il fait largement défaut dans les 
quartiers résidentiels limitrophes, constitue le problème-clef. Le centre-ville doit lui aussi devenir un fleuve ayant peu d'affluents et beaucoup de débouchés, par la création, en bordure, de centres secondaires.

La structure fonctionnelle

La ville - toute ville - est, en dernière analyse, un regroupement de fonctions. Les bâtiments n'en sont jamais que les contenants matériels... Or, ces fonctions ne parviennent à coexister qu'au terme d'une lutte qui a pour enjeu une appropriation du terrain; elles rivalisent d'adresse pour occuper l'emplacement qui leur paraît le meilleur et dont l'acquisition revient finalement à la plus puissante, c'est-à-dire à la plus fortunée. Les prix des terrains et des loyers culminant dans un centre-ville étroit et bondé, il s'opère une hiérarchisation spontanée des fonctions citadines.

121 Celles-ci se partagent en deux larges secteurs: d'une part, le commerce, l'administration et l'industrie; d'autre part, l'enseignement et les activités liées au culte, à la santé, aux loisirs. Entre ces deux secteurs, un trait d'union : l'habitat. C'est là une fonction au vrai sens du terme, c'est même celle qui occupe le plus d'espace dans toute ville. Elle est la clef, l'accès naturel aux fonctions de service, elle en constitue le cadre. Toutefois, elle est de type très particulier: c'est une fonction "négative ", dirions-nous pour la distinguer des fonctions «positives » de production et de services. Nous l'aborderons donc à part, comme une « topographie sociale de la ville », les autres constituant la topographie économique.

Commençons par la fonction commerciale, que nous pouvons diviser en trois types d'activités - en réalité, trois niveaux de centralisation. Tout d'abord, les commerces du centre, qui se livrent une concurrence sans merci au cœur de la ville. Le Cairote peut en sentir le pouls progressivement, depuis les rues al-Galâ' et Ramsès jusqu'à l'extrémité de Midân al-Tahrîr et de Bâb al-Lûq, d'une part, et de la rue al-Gumhuriyya à Midân al-'Ataba, al-Moski et au-delà vers al-Ghuriyya et la rue al-Azhar, etc., d'autre part. Dans ce périmètre se concentrent le négoce de détail et de gros, la circulation de marchandises et de fonds, le commerce moderne et traditionnel. C'est là que siègent les grandes institutions et sociétés, les coopératives, les compagnies d'assurances, les banques, les agents de change, les grands magasins autour desquels s'agglutinent diverses boutiques. Cette zone est le système nerveux, central, de la fonction commerciale pour les habitants de la capitale et des régions satellites.

Notons une importante particularité : le commerce de gros, moins en contact avec le public, a en quelque sorte opéré un retrait et se tient de plus en plus dans les marges de ce quartier, laissant au centre le commerce de détail qui, lui, traite directement avec le public et peut se suffire d'un petit "pied-à-terre »... dont le prix est toutefois exorbitant. Dans les rues al-Galâ' et Ramsès, vers la gare du Caire et la place al-Tahrîr et dans le quartier nommé Ma'rûf, régnent les magasins de gros, proposant surtout des pièces de rechange pour automobiles et des appareils électriques. Sur la place al-Falaki se concentre le commerce des pneux ; celui du papier, du côté de la rue al-Qala'a et à Faggâla, alors que dans la rue al-Gumhuriyya abondent les marchands d'antiquités. Ces rues sont moins fréquentées que les rues du 26-Juillet, Tal'at Harb, Qasr al-Nîl ou celles qui leur sont adjacentes, où l'on ne trouve que le commerce de détail, très dense, débordant de vie et de mouvement. Et alors que le commerce de gros s'octroie des rues ou des quartiers spécialisés, le commerce de détail se répartit selon un mode généralement hétérogène que l'on retrouve surtout dans les grands magasins aux marchandises multiples. 
On trouve ensuite une séparation topographique entre les commerces de type moderne et ceux de type ancien, qui diffèrent aussi par leur clientèle. Celle des premiers est cairote et issue des classes aisées, celle des seconds vient en majorité de la province, de la campagne proche ou lointaine ou bien des couches populaires de la capitale. Le secteur ouest du centre-ville est monopolisé par le commerce moderne; celui de type ancien, le souk, recule vers l'est, part des alentours de la place al-'Ataba, où se trouvent les boutiques populaires traditionnelles. Le souk s'y subdivise en d'autres petits souks; les étalages peuvent quitter les devantures pour les trottoirs. Là aussi les rues se répartissent par spécialités et le commerce de gros y reprend des droits : commerce de maroquinerie, de chaussures et de porcelaine aux angles d'al-'Ataba, d'or et d'orfèvrerie à al-Moski et au souk des bijoutiers, de toile écrue et d'étoffes artisanales dans la rue al-Azhar, d'aromates à al-Ghuriyya...

Il existe également un commerce secondaire ou commerce de quartier établi aux croisements stratégiques dans la plupart des quartiers, sortes de modèles réduits -tels des planètes du système solaire - du centre-ville commercial et à partir desquels se ramifient des filets courant le long des rues principales de la ville. Fermement soudés, solidaires, ces magasins se condensent aux carrefours éloignés du centre.

Reste à évoquer un troisième niveau d'activité commerciale, celui de milliers de boutiques dont l'importance et l'emplacement sont déterminés par la densité de population et par son niveau social. Les quartiers marginaux ou limitrophes au développement récent, comme 'Agûza actuellement, souffrent d'un certain retard : on n'y trouve guère que des produits de première nécessité, des épiceries et coopératives alimentaires, et ils demeurent longtemps mal desservis sur le plan commercial, jusqu'à ce que la population y augmente notablement ; alors s'y installent des commerces plus raffinés.

Abordons maintenant la fonction administrative. L'administration joue un rôle important dans la vie du Caire, capitale politique traditionnellement connue pour son centralisme bureaucratique. Il suffit de savoir que plus du tiers des fonctionnaires s'y concentre. La fonction administrative requiert naturellement des institutions propres, un regroupement topographique et un établissement central, sans qu'il s'agisse nécessairement du cœur encombré et tumultueux de la cité. C'est en bordure du quartier commercial central, vers le sud et le sud-ouest, que se trouvent les principales institutions administratives, suite ininterrompue de bâtisses qui évoquent les casernes d'une armée de fonctionnaires. De Midân al-Tahrîr où se dresse le Mugamma', énorme complexe de services publics indiquant, tel un monument commémoratif, les frontières de cet «État » dans l'État, se déploie comme un seul bloc, entre la rue Qasr al-'Ayni et la ligne de chemin de fer de Hilwân, le quartier des ministères et du Parlement. Mais ce trop-plein déborde ces limites et court jusqu'à la place al-Gumhuriyya, où a longtemps résidé le siège du pouvoir.

De l'autre côté de la rue Qasr al-'Ayni s'articule solidement et directement à ce bloc, tant du point de vue fonctionnel que topographique, une strate spécifique comprenant ambassades et consulats, le palais d'al-Dûbâra et le quartier de Garden City, auquel adhère la chaîne des bâtiments du ministère des Affaires étrangères et de la Ligue arabe. C'est là le territoire d'un autre "État », le corps diplomatique, qui a besoin de traiter directement et immédiatement avec l'« État» voisin, celui des fonctionnaires. Par le passé, au temps du colonialisme, on employait couramment, pour qualifier une relation plus qu'éphémère, l'expression "entre Lazoghli et le palais d'al-Dûbâra " ${ }^{3}$. 
Cependant ce quartier, partiellement lié à la fonction politico-administrative, est essentiellement résidentiel et ne relève pas du centre administratif.

Le Caire, c'est également la capitale de l'industrie égyptienne: on y trouve la plus grande concentration d'usines du pays. L'industrie moderne représente une mutation relativement récente. Si Le Caire est depuis fort longtemps le centre de l'industrie traditionnelle et locale, celle-ci a, aujourd'hui, abandonné la première place à l'industrie moderne. Il nous faut distinguer, d'un point de vue à la fois fonctionnel et topographique, l'industrie légère et l'industrie lourde, l'artisanat manuel, traditionnel - et l'industrie moderne, mécanisée. L'industrie lourde est établie exclusivement à la périphérie de la ville, l'industrie légère en son sein mais loin du centre commercial.

C'est dans un sens relatif et quelque peu abusif que nous avons usé des termes "lourde» et "légère ». Mais eu égard à l'objet de notre étude, il est peut-être acceptable - dans le cadre étroit de la ville - d'appliquer le premier terme aux industries qui ont le plus de " poids » dans l'économie de la ville ou le paysage urbain, et de désigner par le second terme celles de moindre importance, sachant qu'au Caire il ne se trouve d'industries lourdes, au sens propre, que celles du fer et du ciment, installées à Hilwân.

131 L'industrie légère se développe dans les manufactures et ateliers traditionnels de Bulâq et de Sabtiyya; elle consiste surtout en travail de forge, plomberie, réparation et assemblage de machines ; liée à ces activités, la quincaillerie a son marché traditionnel à Wikâlat al-Balah. On trouve aussi, à une échelle réduite, des ateliers de teinturerie et de textile, vestiges de l'activité de grande envergure qui existait au siècle passé, d'où le toponyme d'al-Mabyada ( la blanchisserie ») hérité du blanchiment des tissus écrus qu'on y effectuait au temps de Muhammad 'Ali.

De l'autre côté, à l'est de la ville (derrière al-Moski, al-Ghuriyya, Bâb al-Khalq et jusqu'à Sayyida Zaynab, à Gamaliyya et Darb al-Ahmar) on trouve toute une variété d'ateliers d'artisans et de petites industries traditionnelles et modernes: filatures, industries alimentaires (mise en conserve de fruits, mise en bouteille d'eaux gazeuses), fabriques de verre, ateliers de menuiserie, maroquinerie, tissage, broderie, reliure et autres, installés dans des immeubles bâtis à cet effet ou occupant un étage ou le rez-dechaussée d'immeubles d'habitation. Beaucoup ne correspondent pas aux normes de l'industrie. Il en est de semi-mécanisés qui alimentent le commerce de gros ou de détail.

Ces industries légères, qui ne nécessitent ni de gros capitaux, ni une main-d'œuvre importante, ni une grande quantité de matières premières, et dont les inconvénients bruit, résidus, odeurs - sont relativement supportables, s'intègrent à l'habitat. Mais elles ne peuvent s'établir qu'au sein de quartiers populaires, leur présence limitant nécessairement le standing des habitations voisines. Elles sont cependant une des sources principales de revenu et d'emploi pour leurs habitants, parmi lesquels elles recrutent toute leur main-d'œuvre. Enfin, leur forte concentration est un héritage des activités productives traditionnellement implantées dans ces quartiers. Ces secteurs anciens de l'est de la ville rassemblaient, dans Le Caire médiéval, de multiples organisations, corporations, maîtres artisans. Les industries qu'on y trouve aujourd'hui portent encore la marque de celles d'hier, perfectionnées ou quelque peu décadentes, mais ne relèvent plus d'un territoire spécifique comme c'était le cas par le passé : à l'époque médiévale, chaque métier occupait une impasse, une rue ou un quartier, 
comme en témoignent les toponymes encore usités: al-Surujiyya (fabrication d'huile de sésame), al-Suyufiyya (fabrication d'épées), Sûq al-Silâh (armurerie), al-Mugharbilîn (tamiserie et tamisage), al-Kahkiyyîn (confection de biscuits), al-Fahhâmîn (charbonniers), al-Nahhâsîn (travail du cuivre), etc.

Quant à l'industrie «lourde ", c'est aux confins de la ville que nous la trouvons. C'est une fonction dont le territoire est nécessairement marginal, repoussé aux limites du complexe urbain, voire séparé de lui, aucune logique ne la poussant à se ménager une place en son sein. Bien qu'elle soit beaucoup plus récente, on peut relever les traces anciennes d'ensembles formant, malgré leur peu d'envergure, des concentrations géographiques cohérentes, liées aux spécificités du terrain et rigoureusement en rupture avec le corps de la cité. La taille des pierres, la préparation de la chaux et la briqueterie en offrent quelques, exemples. Toutes les carrières se situent au sud-est du Caire, sur la falaise du Muqattam essentiellement, où elles se succèdent par dizaines, à l'intérieur d'un périmètre qui part des abords de Gabal al-Ahmar et s'étend jusqu'à l'extrémité d'al-Khalifa ; on en trouve également un certain nombre entre les buttes de 'Ayn al-Sirâ et celles de Batn al-Baqara, non loin de Masr al-Qadîma où l'on pratique l'industrie et le commerce de la chaux et du gypse. Ce n'est pas par hasard que beaucoup de bâtiments de l'est du Caire ont été construits en pierre plutôt qu'en briques. Loin des carrières de la montagne, l'industrie de la brique est liée aux îlots du Nil et à leur limon. L'île d'al-Dahab est couverte de fabriques qui sont les premiers fournisseurs de la capitale.

Vers Tûra et al-Ma'sara, nous trouvons des industries dépendant des carrières. Depuis le début du siècle, des installations modernes et de grande envergure, fabriques de ciment et de taille de pierre, ont été érigées à cet endroit. Elles ont proliféré au cours des dernières décennies et ont fini par constituer une concentration des plus importantes dans ce domaine, tant au niveau national qu'au niveau international, leur production couvrant les besoins de la consommation locale et laissant un excédant destiné à l'exportation. Ces deux unités, qui absorbent plusieurs milliers de travailleurs et qui, par leur taille comme par la nature de leur production, comptent parmi les premières dans l'industrie lourde, constituent deux énormes ensembles spécialisés qui, s'ils sont topographiquement en rupture totale avec le corps de la ville, pénètrent toutefois en son cœur et s'infiltrent dans les plus petits interstices du tissu urbain.

En fait, si l'on parle d'industries lourdes, c'est surtout Shubra au nord et Hilwân au sud qu'il faut évoquer. Ce sont là les deux plus grosses zones industrielles de toute l'Égypte. Le montant du capital investi dans chacune d'elles atteint plusieurs centaines de millions de livres, Shubra est la plus ancienne des deux. Ce fut, durant la deuxième guerre mondiale, le terrain des spéculations d'une bourgeoisie étrangère, égyptianisée ou égyptienne qui entendait réaliser des gains importants et rapides dans la filature, le textile et le tricot - les bas de coton en particulier -, activités installées dans des fabriques vétustes, conçues selon un plan anarchique et où régnaient les pires conditions de travail. Le noyau d'abord isolé qui s'était constitué à Shubra al-Khayma se développa avant la nationalisation, puis proliféra par la suite et s'étendit vers le nord, débordant les limites d'al-Qaliubiyya et de la banlieue du Caire, et vers le sud, traversant Shubra al-Mazallât et Shubra al-Balad pour gagner Hadâ'ik Shubra, où il pénétra dans les quartiers habités. Cette industrie passa de la production de cotonnades à celle de lainages, de soieries et de produits synthétiques, développant ses usines de produits complémentaires, de métaux, de pneus, etc., et finissant par constituer une 
zone industrielle variée, jouissant d'une intégration horizontale et verticale au sens propre.

Sous l'impulsion de ce pôle, d'autres noyaux plus modernes virent le jour le long du canal d'Isma'îliyya et de la rue Port-Saïd et se prolongèrent jusqu'à Mastard (mise sous pression du gaz, fabrication de caoutchouc). Autour de ce pôle avaient auparavant surgi des cités ouvrières non planifiées et des bidonvilles qui sont demeurés bien en deçà du seuil de salubrité, constituant un nœud d'encombrement dangereux et rassemblant dans leur périmètre plusieurs centaines de milliers de familles ouvrières.

Liée à cette région industrielle-mère, une excroissance est apparue, moderne et plus modeste: située en face de la première, de l'autre côté du fleuve, dans le quartier d'Imbâba. On y trouve des usines de textile, de cotonnades, de tricot et de bas. Autour de cette zone existe également une cité ouvrière, mais celle-ci est planifiée selon un tracé rectangulaire et a dernièrement attiré dans son voisinage des stations d'alimentation en électricité, en eau, etc.

La logique qui a présidé au choix de l'emplacement de ces zones industrielles suscite sans conteste des interrogations, ceci pour deux raisons : d'une part, elles sont érigées au cœur des terres agricoles et, si elles ont transformé des dizaines de milliers de paysans en ouvriers, elles ont également stérilisé des milliers d'hectares les plus fertiles, sans oublier que leurs résidus sont une dangereuse source de pollution. D'autre part, cette localisation au nord contredit toute logique de planification dans un pays traversé par les vents du nord. S'ils sont vivifiants en hiver et adoucissent le climat en été, ils ont aussi pour effet de rabattre vers le sud les émanations de ces industries, de sorte que le ciel de la capitale en est saturé. Cela suffit sans doute à expliquer le déclin du standing de l'habitat aux abords immédiats de cette zone, et la prolifération d'un habitat moyen et pauvre et de quartiers ouvriers à Shubra, Rôd af-Farag et al-Sâhil, alors que ce secteur, à la jonction de la façade nord et du front fluvial, aurait pu accueillir des quartiers résidentiels. Des raisons économiques ont sans doute entraîné ces choix erronés. C'est à cet endroit que s'effectue la liaison la plus aisée entre la capitale et le Delta, qui lui fournit matières premières, alimentation et accès vers l'extérieur. Les intérêts de la production l'ont emporté sur ceux de l'habitat, tout comme les intérêts des détenteurs de capitaux, avant la nationalisation, ont triomphé de ceux des propriétaires d'immeubles.

140 Il en va différemment au sud. Là, depuis près d'une décennie, l'industrie lourde a envahi une banlieue coupée de la ville - qui était par le passé une banlieue résidentielle et touristique, paisiblement étalée autour de ses sources thermales. Aujourd'hui, les hauts-fourneaux se dressent au voisinage des sources. C'est la première citadelle de l'industrie du fer et du ciment, la base de toutes les industries; elle débuta avec les matières premières des oasis maritimes, transportées par chemin de fer. Au cœur de Wâdi Hôf fut implantée cette forêt d'usines, de cheminées et de hauts-fourneaux qui s'étend sur plusieurs miles et fournit des rails, des wagons, des traverses, des machines, des pièces de rechange, du béton armé, sans oublier la production de voitures - fabrication et assemblage - d'armement, d'appareils ménagers, etc.

141 C'est donc un bouleversement total qui a eu lieu, tant au niveau démographique qu'au niveau économique. Hilwân fait face aujourd'hui à une énorme croissance et va probablement s'étendre jusqu'aux limites du Caire comme elle est entrée dans son orbite économique. Si l'emplacement est ici irréprochable pour ce qui est de préserver 
l'atmosphère de la capitale, il reste que l'avenir de cette station thermale en pleine zone industrielle est difficile à prédire. Il est certain qu'au départ, aucun facteur géographique impératif ne justifiait ce choix si ce n'est le désir de coller à la capitale, ce qui soulève à nouveau îa question de la boulimie de la métropole.

Examinons à présent la fonction des services, à commencer par l'enseignement. Au Caire, la population scolaire est estimée à environ deux millions, soit un cinquième de la population. Les établissements d'enseignement s'imposent donc avec insistance dans le paysage urbain. Leur distribution topographique est organisée par niveaux, de sorte qu'ils dessinent un réseau en forme de grappe, d'arbre ou de pyramide. Les écoles primaires sont les plus répandues et dépendent exclusivement des quartiers d'habitation. Quant aux écoles secondaires, elles relèvent moins du cadre étroit des relations de voisinage que des services de quartier, ce qui explique leur nombre plus réduit et leur éloignement relatif les unes des autres, bien qu'elles dépendent, elles aussi, de l'habitat. Une exception confirme la règle: l'enseignement étranger. Les écoles des communautés et des missions étrangères sont en effet établies non loin du centre commercial de la capitale. (...)

Seul l'enseignement supérieur est organisé en concentrations topographiques nettes, en rupture totale avec l'habitat, liées aux quartiers limitrophes les plus récents et les plus modernes. C'est que l'université a besoin de vastes espaces et de plein air. Ainsi l'université du Caire, bâtie dans la partie moderne de Gîza, s'étend entre Kubri al-Gam'a et Kubri al-Gîza; les bâtiments de l'université de 'Ayn Shams sont dispersés sur une surface allant d'al-Za'farân à 'Abbasiyya. Chacun de ces deux ensembles s'articule sur une arête de la capitale, à l'ouest et à l'est, semblable à des pôles dont la position diverge d'avec ceux de l'industrie - situés au nord et au sud.

Il en va différemment pour l'université d'al-Azhar. Eu égard à son ancienneté et à son caractère religieux, elle se situe dans le quartier le plus ancien de la ville. Elle paie le prix de son histoire comme de sa localisation par l'impossibilité de s'étendre davantage dans ce quartier populaire et dense, qui lui donne son atmosphère particulière. Elle a donc dernièrement opéré une percée en établissant instituts et logements universitaires du côté de 'Abbasiyya. II est intéressant d'évoquer le mouvement historique qui a mené des anciennes universités religieuses aux universités laïques modernes. La mutation survenue au siècle dernier entre les deux types d'enseignement se trouve résumée et symbolisée dans le déplacement qui s'est opéré de l'université d'al-Azhar vers l'université du Caire, soit de l'extrême pointe est de la ville surélevée, archaïque et pauvre, vers son extrême pointe ouest située en terrain plat, moderne et opulent. II est encore plus curieux de constater qu'une étape intermédiaire matérialisée par l'institution de Dâr al-'Ulûm, la faculté de pédagogie et autres instituts voisins et similaires - a transité par le centre de la ville, dans le quartier de Munîra, avant que ces institutions ne soient finalement intégrées aux universités modernes. L'arc décrit par le pendule, allant, en matière culturelle, dans le sens de l'occidentalisation et, sur le plan topographique, vers l'ouest, est ainsi achevé !

En revanche, l'emplacement choisi pour l'enseignement technique, toutes branches comprises, est en rapport avec le lieu où s'exerce tel métier ou avec les quartiers concernés. Généralement, écoles et instituts techniques jouxtent les quartiers industriels. On peut ainsi voir une chaîne serrée d'écoles d'arts et métiers avec leurs ateliers à Bulâq, ancien quartier de l'industrie traditionnelle (industries d'ornementation, vieilles industries mécaniques, ateliers de filage du coton, etc.). Cette 
règle peut, en un sens, s'appliquer à certaines institutions d'enseignement de la médecine dépendant de centres hospitaliers universitaires, où l'enseignement est lié à la pratique. Un des phénomènes les plus frappants réside dans cette constellation polyvalente et dense d'hôpitaux universitaires attachés à la faculté de médecine et aux laboratoires de recherche, concentrée au nord de Rôda et tout le long de Qasr al-'Ayni, de Kubri al-Manial à Fom al-Khalîg, et dont le destin semble avoir été fixé à l'époque de Clôt Bey'. L'idée nous vient immédiatement d'un lien, d'ailleurs réel, mettant en rapport ce périmètre touffu avec la plus grande concentration de médecins et de cliniques de la République, située dans le quartier de Bâb al-Lûq et ses environs; ils n'en sont séparés que par la rue Qasr al-'Ayni.

Passons à une autre fonction qui, contrairement à celle de l'enseignement, est considérée dans une large mesure comme incompatible avec l'habitat, à savoir la fonction sanitaire. Les hôpitaux avec leur vaste surface, leur besoin de calme, les risques de contagion, n'ont généralement pas leur place au cœur des quartiers habités. Et s'il se trouve quelques grands hôpitaux dans la ville même, c'est plus couramment dans les quartiers limitrophes qu'ils élisent leur emplacement, comme c'est le cas pour le grand hôpital public de 'Agûza ou le complexe hospitalier de 'Abbasiyya (psychiatrie, maladies infectieuses et pulmonaires).

D'un certain point de vue, il existe un lien entre les cimetières et la fonction sanitaire, dont l'emplacement doit être rigoureusement choisi. Au sud-est du Caire, traversée par le vent, perchée sur une colline à découvert, s'enracinant dans le sable loin des terres agricoles et coupée du corps urbain, s'élève la cité des morts. Les tombes s'étendent d'al-Ghafîr, au nord, à Imam al-Shâfi'i, au sud, en une bande à peu près ininterrompue délimitée par les carrières de l'est et par le cordon des collines de Qat' al-Mar'a, Zaynhum et 'Ayn al-Sîra à l'ouest.

Toutefois, le quartier d'Imam al-Shâfi'i a amorcé un mouvement rampant vers la cité des morts, la prenant quasiment en chasse. Ainsi la cité des vivants et celle des morts s'enchevêtrent-elles de manière inquiétante. Mais on ne manquera pas de noter ce détail piquant : la seconde est quadrillée de rues bien droites portant des noms et des numéros et offre l'aspect d'un quartier résidentiel pour les morts, et la répartition selon l'appartenance religieuse et le sexe y est évidemment plus rigoureuse que dans la ville des vivants, chaque groupe s'y étant taillé son espace particulier, souverain.

Restent enfin certaines fonctions, semblables, de par leur caractère marginal, à la fonction sanitaire, si ce n'est qu'il ne semble pas en être toujours ainsi au Caire. Les organismes sportifs, terrains de jeux et grands clubs sont par nature des consommateurs excessifs d'espace; et parce que leur public, dans les conditions culturelles et sociales actuelles, se limite aux catégories aisées de la population, ils tendent généralement à s'installer dans les zones résidentielles limitrophes. Ainsi en est-il des clubs Nâdi al-Sayd, al-Zamâlek et al-Tarasâna, situés aux entrées d'al-'Agûza, du stade du Caire à Madinat Nasr, de Nâdi Sibâq al-Khayl (Turf Club) et du Polo Club à Masr al-Gadîda, etc.

On pourrait croire qu'il en est de même pour Nâdi al-Gazîra et Nâdi al-Ahli. Mais le fait est qu'occupant la moitié sud d'al-Gazîra (l'île) pour former la plus grande surface continue consacrée aux loisirs sportifs dans la capitale, les deux clubs sont très proches $\mathrm{du}$ centre-ville, ce qui constitue une exception, une aberration, un anachronisme du point de vue du développement urbain. Nombreux sont ceux auxquels cette critique peut déplaire, mais il faut la comprendre à la lumière du passé. Le colonialisme 
britannique s'était aménagé cet espace, réservé, en outre, à l'aristocratie. C'était dans les premières décennies du siècle, où l'urbanisation de la rive ouest dépassait à peine le centre commercial de Gîza. Les deux clubs se situaient alors effectivement dans des zones limitrophes, en marge de la capitale. Le développement du Caire en général et de la rive ouest en particulier ont eu tôt fait de les noyer, de les intégrer de sorte qu'ils se trouvent aujourd'hui à deux pas du cœur de la ville. De multiples indices montrent qu'ils ont commencé à entraver le développement naturel du centre, et l'affluence de leurs membres constitue en tout cas un facteur saisonnier de perturbation de la circulation. Pire, la stérilité frappe cet espace, interdit l'exploitation idéale d'une énorme surface à la valeur immobilière inestimable, éminemment bien située par rapport à une ville en pleine explosion. Toute planification réfléchie pointe le doigt vers cet emplacement, où elle prévoit soit un quartier résidentiel, soit une zone immobilière à but lucratif (hôtels touristiques,'etc...), soit encore un quartier d'édifices destinés à accueillir conférences, expositions internationales, etc. Une planification raisonnée entraînerait donc ces clubs vers les nouvelles zones limitrophes comme celle de Nâdi al-Sayd. On rétorquera que pareille initiative priverait Le Caire d'un "poumon » naturel ou aggraverait le problème de la densité démographique. Mais l'argument ne convainc pas dans la mesure où c'est le Nil qui, avec ses deux ramifications, constitue ici le poumon naturel adéquat: plus on s'éloigne du fleuve, plus le besoin d'oxygène augmente, particulièrement dans les zones reculées et surpeuplées de la rive est. De plus, Zamalek et Rôda sont bien des quartiers construits et n'ont étouffé personne... Ajoutons à cela qu'aucune capitale au monde, traversée d'un fleuve et ayant en son centre des îles, n'a dédaigné de les exploiter au maximum, et de la meilleure façon possible pour l'habitat. Ainsi en est-il de l'île de la Cité à Paris et de celle de Manhattan à New York.

Les mêmes remarques pourraient s'appliquer à la fonction militaire et à celles de ses institutions siégeant au Caire. Depuis l'époque médiévale, comme en témoigne l'histoire de la Citadelle, la défense a bénéficié d'une cité intégrale et souveraine avec casernes, dépôts et même fabrique d'armements, située à l'extérieur ou en lisière de la ville, du côté est, d'où provient le principal danger externe. À l'opposé, la cité militaire $\mathrm{du}$ temps du colonialisme se situait en plein centre-ville, à Qasr al-Nil, non pour parer au danger extérieur mais pour les besoins internes de l'occupation. Le déplacement du siège de la fonction défensive du sud-est du Caire (La Citadelle) au nord-est ('Abbasiyyaal-Qobba) montre les développements survenus dans le domaine militaire.

152 Il est certain que l'emplacement actuel représente la porte stratégique la plus importante du Caire. Toutefois, on est à nouveau confronté au problème des quartiers limitrophes submergés par le développement de la ville. Le flux démographique a englouti la cité militaire dans sa totalité, débordant son enceinte côté est, en direction du désert. Si pareille situation constitue une entrave au développement urbain, elle contrarie plus encore la fonction militaire elle-même. Cette question avec laquelle ont eu à traiter bien d'autres capitales est maintenant assez mûre pour que l'on puisse envisager de transférer le siège de cette fonction vers les nouvelles limites de la ville.

La topographie sociale

La fonction de l'habitat n'est pas coupée de la topographie sociale; elle en est quasiment le corollaire. La topographie sociale - le terme est emprunté à l'urbaniste français Gaston Bardié - porte principalement sur la répartition des classes sociales dans l'espace urbain. Si la ville socialiste - comme en Union soviétique - ne connaît de 
distinctions topographiques que basées sur la production, les quartiers d'habitation y présentant une homogénéité complète, on ne peut prétendre ici à une "description socialiste » du Caire bien que notre objet d'étude soit la capitale d'un État en voie de socialisation. Au Caire, nous nous trouvons face au résultat d'une longue histoire féodale (iqtâ) et capitaliste, et il nous faudra longtemps encore distinguer les quartiers d'habitation selon les classes économico-sociales. Le logement reste l'expression ultime de la classe ; la maison, c'est le rang, et le quartier exprime la condition sociale.

Il est tout à fait clair que l'ethnie et l'appartenance communautaire sont des aspects secondaires et de bien peu de poids, comparés à la classe sociale; seule celle-ci provoque les changements majeurs, imprime les traits les plus distinctifs de la topographie sociale de l'ancienne et noble capitale d'un peuple dont l'unité et la cohésion sont un fait reconnu depuis des millénaires. C'est l'opposé dans la ville américaine, privée d'histoire, ville d'émigrants caractérisée essentiellement par l'hétérogénéité ethnique, la disparité des races et des nationalités: la race y a une dimension non moins importante que la, classe dans la morphologie sociale.

En simplifiant, on peut localiser les quartiers pauvres aux extrêmes sud, est et nord de la ville ainsi qu'en son milieu, où l'on repère une étendue formant comme une grande île. À l'extrême sud, dans certaines parties du centre commercial de Gîza, des parties de Masr al-Qadîma jusqu'à Sayyida Zaynab via Abû-l-Su'ûd, al-Madâbigh (les tanneries), alMazbah (l'abattoir) et al-Bagghâla (les muletiers). À la pointe est, d'al-Khalifa à alHusayniyya via al-Qala'a, al-Darb al-Ahmar et Gamaliyya. À l'extrême nord, dans les zones périphériques de Shubra al-Khayma, Shubra al-Balad, al-Sâhil, leurs environs et prolongements via Mastard, Mahmasha et al-Shamâshargi puis, en face, Imbâba. Quant à l'îlot du milieu, il est formé par le bloc de Bulâq-al-Sabtiyya ; on trouve parfois des poches secondaires dans les environs du quartier construit sur la rive ouest : ce sont des villages ou des cités ouvrières englouties par le développement urbain comme Bulâq al-Dakrûr et Bayn al-Sarâyàt.

Il apparaît clairement que ce sont soit des quartiers populaires très anciens, dont les bâtiments vétustes sont parfois proches de l'effondrement, les rues non planifiées, la densité d'habitations et d'habitants élevée, les familles nombreuses, soit des quartiers ouvriers de construction récente mais de bas standing, où l'on peut trouver des fractions de la petite bourgeoisie, petits fonctionnaires et artisans. Encore plus manifeste est cette interpénétration, plus ou moins importante, de l'industrie et du commerce que nous avons déjà signalée. Enfin, la plupart de ces quartiers se situent en terrain élevé.

157 À l'opposé, les riches quartiers résidentiels occupent la plus grande partie des terrains les plus proches du fleuve sur la rive ouest, au nord du centre commercial de Giza, et la plus grande partie également de l'île de Rôda et de l'île de Zamâlek. Puis l'on traverse pour arriver à Garden-City et Qasr al-Dûbâra. Plus loin encore, nous trouvons Masr alGadîda et de nombreux quartiers du nord-est, à partir d'al-Qubba. Le facteur géographique commun à tous ces quartiers, à l'exception de Masr al-Gadîda et de ses environs, c'est leur situation en terrain bas, sur le front fluvial.

Dans l'ensemble, ces quartiers sont réservés à l'habitai ; si d'autres fonctions y font leur apparition, ce sont certaines institutions administratives, ministères ou services publics mais surtout, des missions diplomatiques : ambassades et consulats abondent à Garden City, Qasr al-Dûbâra, Zamâlek, Doqqi et, depuis une époque récente, à 'Agûza. $\mathrm{Si}$, à la présence d'ambassades et de missions diplomatiques, correspond un haut 
standing de logements, ce n'est que dans les quartiers résidentiels relativement proches du centre-ville. Les bâtiments à caractère diplomatique sont absents des quartiers éloignés comme Masr al-Gadîda.

Ce sont les hauts immeubles, parfois de petits gratte-ciel, qui dominent ici le paysage urbain ; les bâtiments y sont toujours modernes, de construction récente. Les villas y sont peu nombreuses en raison du coût très élevé des terrains pris sur la terre agricole, d'où la nécessité d'exploiter intensivement l'espace vertical pour y garantir une densité maximale. Garden-City ne mérite pas son nom, et l'idée de «cité jardin » connue en Europe depuis Howard y est même tournée en dérision. C'est une forêt d'énormes immeubles qui se dresse dans ce quartier, plutôt qu'une constellation de villas noyées dans une mer de verdure. La villa reprend ses droits, cependant, sur les sables de Masr al-Gadîda et des banlieues nord-est, où l'on peut goûter le luxe d'espaces horizontaux. Ces quartiers sont le lieu de prédilection des classes dirigeantes aux revenus les plus élevés. Après la Révolution, une opération de "succession domiciliaire » est survenue, entraînant un changement de résidants. C'est dans ces quartiers que les minorités européennes coloniales avaient préférentiellement élu domicile, de même qu'y étaient installées les familles de capitalistes et d'industriels autochtones. Avec la liquidation des uns et des autres, s'y est progressivement installée une petite bourgeoisie aisée, intellectuelle, contribuant dans une certaine mesure à adoucir le relief de la topographie sociale dans la capitale.

160 Entre les deux opposés, quartiers pauvres et quartiers riches, se glissent les quartiers moyens par leur position géographique ou sociale, habités le plus souvent par des fonctionnaires, des intellectuels ou des commerçants de la classe moyenne. Outre sa présence à l'arrière-plan de la rive ouest, cette classe domine à Fomm al-Khalîg, devient souveraine dans le quartier de Munîra, ses environs et plus en arrière, jusqu'à la limite des quartiers modestes situés à l'est de la ville; elle est à nouveau majoritaire dans toute la zone s'étendant en largeur, depuis al-Faggâla, al-Dhâhir, Ghamra et al-Sakakîni Jusqu'à al-Wâyli et al-'Abbasiyya, puis dans de grands secteurs de la banlieue nord-est, sans compter le secteur le plus important, au sud, de Shubra et Rôd al-Farag. Les voies ferrées des lignes nationales ou de banlieue traversent généralement ces quartiers d'habitat moyen ou pauvre, polluant sur toute leur longueur les régions attenantes et abaissant leur niveau social.

161 Que signifie cette carte sociale ? Les rapports qui président à la répartition des trois classes dans l'espace urbain ont-ils un sens?

L'élément le plus frappant consiste dans le principe de cloisonnement qui commande généralement l'habitat : à chaque classe son quartier, à chaque quartier sa classe. La rupture est cependant "pacifique ", en ce sens que d'un quartier à l'autre, les classes s'échelonnent comme elles le font dans la société. En d'autres termes, il est rare que les quartiers des classes riches et ceux des classes pauvres soient immédiatement juxtaposés; le plus souvent s'immiscent entre eux des quartiers habités par la classe moyenne comme c'est le cas dans le centre, sur l'axe Garden City/al-Munîra/al-Qala'a.

Il arrive toutefois que ces deux classes se rapprochent, se retrouvent face à face. Dans certains cas, c'est même inévitable étant donné l'importante force de travail que l'une puise dans l'autre pour son service domestique. Mais alors, elles sont nécessairement séparées par un obstacle naturel, tel le Nil entre Zamâlek et Bûlaq, qui met en évidence l'antinomie sociale parvenue à son plus haut degré, cependant que la distance géographique est réduite au minimum, ou entre Rôda et Masr al-Qadîma, bien que de 
façon moins tranchée... En ce qui concerne les normes sous-jacentes qui régissent cette disposition, on peut s'interroger sur le facteur de proximité ou d'éloignement du centre-ville. Dans beaucoup de villes européennes ou américaines, la distance du logement au centre est devenue la norme obligée pour l'évaluation du niveau social, de l'appartenance de classe. Plus la distance est grande, plus le niveau social est élevé et vice-versa. Le Caire n'obéit que partiellement à cette règle (Masr al-Gadîda, al-Ma'âdi) et même la contredit le plus souvent (Garden City, Zamâlek d'un côté, Imbâba, Shubra al-Khayma et Masr al-Qadîma de l'autre).

La haute ou basse altitude du terrain constitue une autre norme de répartition sociale dans les villes froides d'Occident, où les basses terres sont des pièges à brouillard alors que les terres élevées sont salubres, sèches, exposées à la lumière, de sorte qu'aux hautes positions sociales correspondent les positions géographiques élevées et aux humbles positions sociales correspondent les positions géographiques de basse altitude. $\mathrm{Au}$ Caire, la situation contrevient en grande partie à cette règle. L'est de la ville, le plus élevé, abrite les quartiers pauvres, ouvriers, populaires alors qu'à l'ouest, sur les terres basses du bord du Nil, dans ses îles et sur sa rive ouest se concentrent les riches habitations, avec une exception de taille : le secteur de Bûlaq et, au nord, Shubra alKhayma, ses environs et Imbâba ; ce sont toutes des terres basses avec des quartiers modestes et pauvres.

165 La direction des vents serait-elle donc la norme? On a remarqué qu'en Occident, les beaux quartiers avaient tendance à monopoliser la partie ouest de la ville, ouverte aux vents frais et non pollués venant de cette direction.

166 En Égypte, il ne fait pas de doute que l'on prise fortement les vents maritimes, ce qui se traduit dans le prix des terrains et des loyers; de même, les beaux quartiers des villes moyennes sont attirés vers le nord à la manière de l'aiguille aimantée d'une boussole. Au Caire, on se heurte toutefois au quartier industriel de Shubra et à Imbâba alors que Masr al-Gadîda et les banlieues nord-est sont à découvert, aucun obstacle ne s'interposant entre elles et les vents maritimes.

167 L'attrait du fleuve constitue encore une norme possible. Les beaux quartiers sont inévitablement attirés par le front fluvial, vivifiant dans un climat chaud, comme par la vue donnant sur ce paysage naturel. Il est clair que ce facteur se reflète largement sur le terrain au Caire ; il n'y a qu'à considérer la plus grande partie de la rive ouest, les deux îles et Garden City. Cependant, il ne traduit pas toute la réalité : Bulâq et Imbâba sont situés sur le fleuve. Masr al-Gadîda s'en trouve on ne peut plus loin. Cela ne diminue pas pour autant l'importance de ce facteur puisqu'à l'intérieur des régions habitées par une même classe sociale, qu'elle soit aisée ou moyenne, les meilleurs logements sont généralement situés sur le front fluvial et leur qualité s'échelonne selon qu'ils s'en éloignent ou s'en rapprochent. Sur la rive est, par exemple, le niveau du logement présente une baisse régulière au fur et à mesure que l'on s'éloigne du fleuve. Il n'y a donc pas de clé unique. On peut toutefois avancer que le front fluvial l'emporte sur les vents qui, à leur tour, prennent l'avantage sur le relief.

Telle est donc la configuration de la société cairote dans son foyer géographique, son milieu naturel. Si la classe sociale en détermine les principaux traits, les minorités en complètent l'image, la précisent par d'ultimes touches sans empiéter sur les coups de brosse initiaux. D'importants changements sont survenus au cours de la dernière décennie en ce qui concerne le volume et la répartition des minorités étrangères et des colonies européennes en raison de «l'exode blanc » qui a accompagné l'indépendance. 
Mais avant cette date, celles-ci ont longtemps pesé dans la balance car elles comptaient des dizaines de milliers d'âmes, même si elles étaient moins nombreuses qu'à Alexandrie.

Dans les années trente et quarante, la répartition des Européens au Caire était principalement caractérisée par leur regroupement dans la moitié nord de la capitale ou plutôt, par leur absence totale dans la partie sud. Leur position au nord était assez proche du centre-ville, avec de fortes concentrations à Garden-City et Qasr al-Dûbâra, puis autour de Midân al-Isma'îliyya et Tawfîqiyya: dans de nombreux secteurs, ils constituaient plus de la moitié des habitants. Dans le périmètre s'étalant entre alFaransâwi et Bâb al-Lûq, et entre Ghamra et Shubra, leur pourcentage variait entre la moitié et le cinquième des habitants.

Cette répartition met en évidence la tendance naturelle des minorités étrangères au regroupement et leur gravitation - peu courante chez les autochtones mais logique pour les étrangers - vers le centre-ville commercial où ils associent travail et logement, et où apparaissent également les hôtels, pensions, etc. De plus, la répartition des minorités étrangères s'inscrit dans le cadre général de la disposition par classes sociales. Les éléments les plus riches et les plus influents se retrouvaient dans les beaux quartiers de Garden City et de Zamâlek, ceux d'un moindre rang social dans les quartiers de la moyenne bourgeoisie, mais dans tous les cas, ils étaient résolument éloignés des quartiers pauvres. Certaines colonies ont été, traditionnellement ou occasionnellement, liées à certains quartiers : les Anglais à Garden City et Zamâlek, ou dans la banlieue isolée de Ma'âdi; les Grecs, Italiens et Levantins aux entrées de Shubra, vers la gare (ceux de Bilâd al-Shâm à Qusûrat al-Shawâm en particulier).

Enfin, l'isolement de ces communautés par rapport à la population indigène n'a jamais été aussi strict que dans les capitales coloniales d'Afrique ou d'Asie. Certains Européens, parmi les moins fortunés, se sont complètement intégrés aux blocs de peuplement autochtones; de plus, la ville coloniale fermée n'a jamais vu le jour en Égypte. Les Anglais, forts de leur autorité coloniale et avec une arrogance toute anglosaxonne, ont bien tenté de se confiner dans la banlieue de Ma'âdi, édifiée pour leur compte - séparatisme masqué par l'isolement naturel du lieu - mais ils ont échoué : leur banlieue a été prise d'assaut par des éléments indigènes. De tout cela, il ressort que l'écart culturel et ethnique entre Européens et Égyptiens a toujours été vécu d'une manière différente de celle qui avait cours dans de nombreuses colonies du TiersMonde; le colonialisme n'a jamais pu établir en Égypte la moindre «barrière de couleur ».

172 En ce qui concerne l'aspect religieux, les communautés européennes concentrées dans le centre-ville commercial ou à proximité ont édifié leurs institutions religieuses dans ces mêmes zones et non dans les quartiers d'habitation, comme c'est la tradition pour les institutions religieuses du pays. Après le départ de ces communautés, leurs édifices religieux sont demeurés en place, comme la cathédrale anglicane à Maspero, la cathédrale Saint-Joseph, de nombreuses églises à Bâb al-Lûq, la synagogue de la rue 'Adli, etc. 


\section{NOTES}

1. CLERGET M., Le Caire, Étude de géographie urbaine et d'histoire économique, Le Caire, 1934 (2 vol.).

2. En ce qui concerne l'Égypte notamment, le mot « féodalité » est en réalité impropre pour traduire iqtâ', qui évoque un système tout à fait différent de ceux qui existaient en Europe (N.D.T).

3. Lazoghli était à l'époque le siège des fonctionnaires d'État et Qasr al-Dûbâra était occupé par le ministère des Affaires étrangères (N.D.T).

4. Clôt Bey : médecin français que Muhammad 'Ali fit venir en Égypte pour diriger

l'école de médecine militaire. Il dirigea en outre l'hôpital al-Qasr al-'Ayni (N.D.T.).

INDEX

Mots-clés : Le Caire, Grand Caire 